\title{
Metagenomic next-generation sequencing for identifying pathogens in central nervous system complications after allogeneic hematopoietic stem cell transplantation
}

\author{
Wenjun Liu ${ }^{1}$ Zhiping Fan ${ }^{1}$ Yan Zhang ${ }^{1} \cdot$ Fen Huang ${ }^{1} \cdot \mathrm{Na} \mathrm{Xu}^{1} \cdot \mathrm{Li} \mathrm{Xuan}^{1} \cdot \mathrm{Hui}^{\mathrm{Liu}}{ }^{1} \cdot$ Pengcheng Shi $^{1} \cdot$ \\ Zhixiang Wang ${ }^{1} \cdot$ Jun $\mathrm{Xu}^{1} \cdot$ Xiaofang $\mathrm{Li}^{1} \cdot$ Jing Sun ${ }^{1} \cdot$ Qifa Liu ${ }^{1}{ }^{1} \cdot \operatorname{Ren} \operatorname{Lin} \mathbb{D}^{1}$
}

Received: 30 October 2020 / Revised: 26 January 2021 / Accepted: 12 February 2021 / Published online: 6 April 2021

(c) The Author(s), under exclusive licence to Springer Nature Limited 2021

\begin{abstract}
A prospective study was conducted to compare metagenomic next-generation sequencing (mNGS) and conventional testing in investigating the pathogens of central nervous system (CNS) infections in allogeneic hematopoietic stem cell transplantation (allo-HSCT) recipients. A total of 53 patients with CNS disorders after allo-HSCT were enrolled in this study. A total of 35 patients were diagnosed as CNS infections, including 28 viral, 2 bacterial, 1 fungal, 3 mixed infections, and 1 infection with unknown pathogen. Among these 35 patients with CNS infections, mNGS identified 5 patients who were not identified by conventional testing. For the remaining 30 infections, mNGS made concurrent diagnoses with conventional testing in 29 , while 1 was diagnosed according to the good response to the antimicrobial treatment without etiological evidence. The presence of Aspergillus detected by mNGS only in one patient was considered false positive due to lack of validation. The sensitivity of mNGS and conventional testing for diagnosing CNS infections post transplant were $97.1 \%$ and $82.9 \%$, respectively $(P=0.106)$, while the specificity of mNGS and conventional testing were $94.4 \%$ and $100 \%$, respectively $(P=1.000)$. These results suggest that mNGS might be a promising technology for diagnosis of CNS infections post transplant. Viruses were the most common pathogens of CNS infections in allo-HSCT recipients.
\end{abstract}

\section{Introduction}

Allogeneic hematopoietic stem cell transplantation (alloHSCT) is a curative option for a wide range of disorders such as hematological malignancies and some nonmalignant diseases. Central nervous system (CNS) infections are severe complications with high mortality in allo-HSCT recipients [1-3]. The incidences of CNS infections after

These authors contributed equally: Wenjun Liu, Zhiping Fan,

Yan Zhang

Supplementary information The online version contains supplementary material available at https://doi.org/10.1038/s41409021-01243-8.

Qifa Liu

liuqifa628@163.com

$\triangle$ Ren Lin

lansinglinren@163.com

1 Department of Hematology, Nanfang Hospital, Southern Medical University, Guangzhou, China
allo-HSCT differed widely, which ranged from 1.2 to $15 \%$ [4-12]. The pathogens of CNS infections also varied among the studies [5, 6, 11, 13-17]. These discrepancies are partly attributed to the different testing methods. Conventional testing, such as smear microscopy and culture, remains commonly used to identify pathogens in clinical settings, but these are relatively insensitive and mainly used for the detection of bacteria, fungi, as well as parasites [18]. Although serologic testing has been widely used to identify viruses, it is unfeasible for most of transplant recipients because of immunosuppression [19]. Recently, nucleic acid amplification techniques including real-time quantitative polymerase chain reaction (RQ-PCR) and multiplex PCR have been widely used in diagnosis of infectious diseases, especially viral infections. It has been recognized as a "gold standard" to identify viruses in CNS infections [1]. Nevertheless, the use of RQ-PCR is still limited by the genetic diversity of some pathogens families and requirement of a prior knowledge on the target as well as the low pathogens loads and limited sample volumes in cerebrospinal fluid (CSF). Therefore, novel diagnostic methods are urgently needed to detect all the potential pathogens from undetermined types of CNS infections. 
Metagenomic next-generation sequencing (mNGS), as an emerging approach with the ability to detect all potential pathogens in a single assay, is attracted more and more attention [20, 21]. However, the clinical usefulness of mNGS remains under discussion in diagnosis of CNS infections after allo-HSCT. In this study, we compared mNGS and conventional testing in investigating the pathogens of CNS infections, especially viruses, in allo-HSCT recipients.

\section{Materials and methods}

\section{Patients and study design}

Patients who underwent allo-HSCT were enrolled in this prospective study if they met one of the following criteria: (1) patients presenting with CNS disorders following systemic viruses-associated diseases or viremia, and (2) patients presenting with CNS disorders without a diagnosis at time of enrollment. CNS disorders attributed to previous established noninfectious causes (i.e., drug-related toxicity, electrolyte abnormality, and continued CNS involvement of primary disease) were excluded. Methods for detecting potential pathogens included conventional testing and mNGS. Pathogens detected by mNGS only were further confirmed by Sanger sequencing, PCR, or treatment response.

This study was performed in accordance with the modified Helsinki Declaration, and the protocol was approved by the Ethics Committee of Nanfang Hospital before study initiation. All the recipients and/or guardians provided written informed consent prior to study enrollment.

\section{Infection prophylaxis and virus monitoring}

Infection prophylaxis was performed as previously described $[22,23]$. The Epstein-Barr virus (EBV) and cytomegalovirus (CMV) DNA in the peripheral blood (PB) were measured by RQ-PCR weekly for the first 3 months and once every 2 weeks from the 4th to the 9th month, then once per month from the 10th to the 12th month after transplantation. The threshold for EBV/CMV-DNA copies in plasma provided by the manufacturer (ZJ Bio-Tech Co., Ltd., Shanghai, China) was 500 copies $/ \mathrm{mL}$. Immunosuppressants were reduced whenever feasible in the patients with CMV-DNAemia or EBV-DNAemia. Ganciclovir or foscarnet was used for the preemptive therapy of CMV-DNAemia and rituximab for EBV-DNAemia [22]. Rituximab-based preemptive therapy was administrated as follows [23]: (1) EBV DNA in blood was positive twice consecutively with a rising trend over 1 log; (2) EBV DNA was positive consecutively six times without a dropping trend over $1 \log$; or (3) EBV DNA was positive consecutively eight times. To the patients who showed noncomplete response after one cycle of rituximab treatment, the adoptive cellular immunotherapies following rituximab, including donor lymphocyte infusion or EBVspecific cytotoxic T lymphocytes, were used. Other viruses were not routinely monitored and were only tested when patients presented with specific symptoms and were suspected of viral infections.

\section{Etiological assessment}

Etiological assessment included clinical, imaging, laboratory, and pathogens findings. The patients enrolled all received computed tomography (CT) or/and magnetic resonance imaging (MRI) examination, and lumbar punctures within $72 \mathrm{~h}$ after symptom onset. The methods for pathogens detection in CSF included conventional testing and mNGS. Conventional testing included smear microscopy for bacteria, fungi, and parasites, culture for bacteria and fungi, RQ-PCR for viruses, mycoplasma pneumoniae, chlamydia pneumoniae, and toxoplasma. RQ-PCR were performed in accordance with the manufacturer's protocol and the assessed viruses included EBV, CMV, herpesvirus 6-8 (HHV-6-8), herpes simplex virus 1 and 2 (HSV-1-2), varicella zoster virus (VZV), JC virus, adenovirus, human rotavirus, human bocavirus, influenza A virus (FluA), influenza B virus (FluB), parainfluenza virus (PIV1, PIV2, PIV3, PIV4), respiratory syncytial virus (RSV), human metapneumovirus, human coronavirus (229E, OC43, NL63, HKU1), and enterovirus (Guangzhou HuYanSuo Medical Technology Co., Ltd) [24]. mNGS was performed by the Beijing Genomics Institute according to the manufacturer's protocol [25]. The detailed process and positive criteria for NGS are shown in Supplementary Appendix.

\section{Diagnosis of CNS infections}

The diagnosis of CNS infections was according to the related criteria [1]. In brief, proven CNS infection was diagnosed as following: (1) neurological manifestations with normal or abnormal neuroimaging; (2) identification of pathogens in CSF; and (3) histopathological evidence if necessary. Probable CNS infection was established based on neurological manifestations, absence of other noninfectious etiologic evidence or established diseases, and good response to the antimicrobial treatment. EBVassociated posttransplant lymphoproliferative disorders (PTLD) was classified as EBV infection.

\section{Statistical analysis}

Quantitative variables were expressed as medians (ranges) and qualitative variables were expressed as percentages. The Chi-square or the Fisher exact test was used to compare the sensitivity and specificity between mNGS and conventional testing related to the final diagnosis. The incidence of 
CNS infections was estimated as cumulative incidence, considering death without CNS infections as a competing event. All $P$ values were two-sided with significance level at $\alpha=0.05$. The SPSS statistics 19.0 (SPSS, Chicago, IL) and $\mathrm{R}$ version 3.4.3 (R Development Core Team, Vienna, Austria) were used for data analysis.

\section{Results}

\section{Patients' characteristics}

A total of 53 in the 567 patients undergoing allo-HSCT between July 2016 and March 2019 were enrolled in this prospective study. A total of 31 patients were males and 22 were females, with a median age of 28 years (range, 16-67). Primary diseases included acute myeloid leukemia (AML, $n=34$ ), acute lymphoblastic leukemia (ALL, $n=11$ ), myelodysplastic syndrome $(n=4)$, chronic myeloid leukemia (CML, $n=2)$, and primary myelofibrosis $(n=2)$. A total of 14 patients received HLA matched and 39 received HLA-mismatched donor transplantation. All the patients received myeloablative conditioning regimens. Antithymocyte globulin was administrated in 37 patients for GVHD prophylaxis.

\section{Pathogens detected in CSF samples}

Of the 53 patients enrolled, 38 pathogens were detected in 34 patients including viruses $(n=33)$, bacteria $(n=3)$, and fungi $(n=2)$. A total of 32 pathogens were identified by mNGS and conventional testing both and 6 by mNGS only. The pathogens detected by both mNGS and conventional testing included EBV $(n=10)$, HHV-6 $(n=9)$, adenovirus $(n=3), \mathrm{HSV}-1 \quad(n=3), \mathrm{CMV}(n=3), \mathrm{VZV}(n=2)$, Aspergillus $(n=1)$, and Enterococcus $(n=1)$. The pathogens detected by mNGS only included EBV, HHV-7, HSV1, Streptococcus pneumoniae, Rickettsia felis, and Aspergillus. A total of 19 patients had elevated CSF pressure (median, $2.16 \mathrm{kPa}$; range, $1.96-2.65 \mathrm{kPa}$ ). A total of 17 patients had high CSF protein (median, $1.01 \mathrm{~g} / \mathrm{L}$, range, $0.46-1.91 \mathrm{~g} / \mathrm{L}$ ). Five patients had pleocytosis (median, 104/ $\mu \mathrm{L}$; range $10-300 / \mu \mathrm{L}$ ). Blast cells were found in CSF in all the six patients with CNS leukemia, while atypical lymphocytes were observed in CSF in the two patients who were diagnosed as CNS-PTLD. The CT or MRI of the CNS was abnormal in 25 patients including focal lesions in 16 and diffuse changes in 9 patients.

\section{Validation for the pathogens}

Among the pathogens detected by mNGS only, viruses were confirmed by validation testing via Sanger sequencing.
Bacteria were further confirmed by the good response to the antimicrobial treatment. Aspergillus was considered false positive for lack of validation via PCR as well as galactomannan detection in CSF sample. Besides, the patient recovered after the supportive treatment without antimicrobial agents. Finally, the diagnosis of CNS infections was established in five of the six patients with positive findings of mNGS only. In addition, one patient with increased CSF pressure and protein content but lack of positive finding by either mNGS or conventional testing was cured by a combination of empirical antibacterial and antifungal therapy, and then was diagnosed as CNS infection. Ultimately, 35 patients were diagnosed CNS infections post transplant. The spectrum of pathogens is shown in Table 1, and viruses were the most frequent causes. The sensitivity of mNGS and conventional testing for diagnosing CNS infections post transplant were $97.1 \%$ and $82.9 \%$, respectively $(P$ $=0.106)$, while the specificity of mNGS and conventional testing were $94.4 \%$ and $100 \%$, respectively $(P=1.000)$.

\section{Etiology of CNS complications}

Etiology was identified in 46 of the 53 patients enrolled including CNS infections in $35(66.0 \%)$ and noninfectious causes in $11(20.8 \%)$ patients. Noninfectious causes included CNS leukemia $(n=6,11.3 \%)$, Bell's palsy $(n=2$, $3.8 \%)$, demyelinating syndromes $(n=2,3.8 \%)$, and

Table 1 The spectrum of pathogens in CNS infections.

\begin{tabular}{lr}
\hline Pathogens & Cases \\
\hline Virus & 28 \\
EBV & 10 \\
HHV-6 & 8 \\
HSV-1 & 3 \\
Adenovirus & 3 \\
VZV & 2 \\
CMV & 1 \\
HHV-7 & 1 \\
Bacteria & 2 \\
Streptococcus pneumoniae & 1 \\
Rickettsia felis & 1 \\
Fungi & 1 \\
Aspergillus & 1 \\
Mixed infections & 3 \\
Enterococcus + CMV & 1 \\
HHV-6 + EBV & 1 \\
HSV-1 + CMV & 1 \\
Unknown & 1 \\
\hline
\end{tabular}

$E B V$ Epstein-Barr virus, $H H V$-6 herpesvirus 6, $H S V$-1 herpes simplex virus $1, V Z V$ varicella zoster virus, $C M V$ cytomegalovirus, $H H V-7$ herpesvirus-7. 
posterior reversible encephalopathy syndrome $(n=1$, 1.9\%). CNS leukemia included AML relapse with CNS involvement $(n=3)$, ALL relapse with CNS involvement $(n=2)$, and CML in lymphoid blast crisis relapse with CNS involvement $(n=1)$. Etiology of seven patients $(13.2 \%)$ remained unclear.

\section{Discrepancy of virus between CSF and PB}

The viruses' DNA/RNA loads were compared between the CSF and PB sampling simultaneously in the patients with CNS viral infections. Virus was detected in CSF in four patients (HHV-6 in two and EBV CNS infections in two), while no virus was detected in $\mathrm{PB}$ at that time.

\section{Treatment and outcome}

A total of 50 patients, including 34 CNS infections, 11 noninfectious CNS complications, and 5 undetermined, received treatment. Three patients abandoned treatment because of the patients' desire and financial constraint. Treatments were given based on the etiology, and supportive treatments were given for those with unclear diagnosis. Of the patients with CNS infections, 24 had good response to the treatments and survived, while 9 died because of CNS infections progressing and 1 died of severe pneumonia although with improving CNS disorders on the third day of treatment. Ten of the patients with noninfectious CNS complications achieved good response during the treatments, while one died of CNS leukemia relapse. Two of the patients with unclear diagnosis had good response to the treatments which included antiemetic, neurotrophic, and antipyretic analgesics, while three died because of acute pancreatitis, multiple organ failure, and thrombotic microangiopathy in each.

\section{Cumulative incidence of CNS infections}

The median onset time of CNS infections and CNS viral infections were 54 days (range, 6-733) and 52 days (range, 6-733) post transplantation, respectively. The 1-year and 2year cumulative incidence of overall CNS infections post transplants were $5.51 \%$ (95\% confidence interval (CI), $3.83-7.61 \%$ ) and $6.59 \%$ (95\% CI, 4.65-8.96\%), respectively. The 1-year and 2-year cumulative incidence of CNS viral infections post transplants were 4.45\% (95\% CI, 2.96-6.38\%) and $5.29 \%$ (95\% CI, 3.57-7.48\%), respectively.

\section{Discussion}

mNGS provides a comprehensive method, by which nearly all potential pathogens can be accurately identified, in a single method without the need for sequence-specific amplification. Miller et al. presented $73 \%$ sensitivity and 99\% specificity of mNGS for pathogens identification in 95 CSF samples of immunocompetent patients with meningitis and encephalitis [26]. Miao et al. reported that mNGS had a higher sensitivity ( $52.5 \%$ vs $34.2 \%$ ) and a similar specificity (85.7\% vs $89.1 \%$ ) compared with culture among immunocompetent individuals with acute or chronic infections [20]. The primary objective of the current study was to evaluate the clinical usefulness of mNGS in identifying the pathogens of CNS infections, especially CNS viral infections, in a setting of patients with CNS disorders after allo-HSCT. Our results showed that the sensitivity of mNGS and conventional testing in diagnosis of CNS infections post transplant were 97.1 and $82.9 \%$, while the specificity of mNGS and conventional testing were 94.4 and $100 \%$. mNGS seemed to have a trend of higher sensitivity and a similar specificity to identify the pathogens of CNS infections in allo-HSCT recipients compared with conventional testing. Of interest, the sensitivity of mNGS in the current study was higher than those in the previous studies [20,26]. We assumed that the discrepancies might be attributed to the different enrolled cohorts and reference standards, as well as the different type of infectious diseases. The patients enrolled in the current study were suspected of CNS infections, especially viral infections in which mNGS was considered sensitive $[20,26]$. Besides, we used the clinical diagnosis as the reference standard instead of the results of conventional testing which might be incorrect in some cases $[20,26]$. These results need to be further explored with large sample-size studies.

The incidence and spectrum of CNS infections varied in the available studies which were mainly retrospective [413, 27-31]. The current prospective study showed that the 2-year cumulative incidence of overall CNS infections and viral CNS infections were $6.59 \%$ and $5.29 \%$, respectively, which were consistent with our previous prospective study [27]. In respect to the spectrum of CNS infections after allo-HSCT, early studies showed that toxoplasma and fungi were the main pathogens of CNS infections [5, 6, 11, 13, 14]. A retrospective study enrolled 655 HSCT recipients between 1990 and 1997 reported that the most common pathogen was toxoplasma followed by Aspergillus, Candida, and viruses [6]. Over the decades, with the wide use of prophylactic broad-spectrum antifungal agents and novel testing methods, a growing body of studies suggested that viruses, especially herpesvirus, had become main causes of CNS infections after allo-HSCT $[3,12,15,16,27]$. Similarly, viruses were found as the most common pathogens in the current study, followed by bacteria and fungi. As to the kinds of viruses, many studies demonstrated that herpesviruses were most frequent $[12,27,32]$. EBV was found the leading cause of CNS viral infections in the current study, which consisted with the 
results of the two previous studies including ours [16, 27]. However, some retrospective studies revealed that HHV-6 was the most frequent $[3,4,12,32]$. Moreover, a retrospective study from China suggested that RSV was the most common cause of CNS viral infections in haplo-identical HSCT recipients [17]. A reasonable interpretation of these differences was that EBV-associated CNS-PTLD was classified as CNS infectious disease in our studies.

Viremia was historically considered important for the diagnosis of herpesvirus-associated diseases, but emerging evidences suggested that the discrepancy of viruses, especially herpesvirus, between CSF and PB in CNS infections was not rare [33-35]. This phenomenon was also observed in our study. Therefore, CNS viral infection should not be excluded in the patients who had no viremia.

In summary, mNGS might be a promising technology for diagnosis of CNS infections post transplant. Viruses were the most common pathogens of CNS infections in alloHSCT recipients.

Acknowledgements This work was supported by grants from the National Key Research and Development Projects (Grant Nos 2017YFA0105500; 2017YFA0105504), the National Natural Science Foundation of China (Grant Nos 81770190, 81970161), the Key research and development plan of Guangdong Province (Grant No. 2019B020236004), and the Science and Technology Program of Guangzhou (Grant No. 201707010213).

\section{Compliance with ethical standards}

Conflict of interest The authors declare no competing interests.

Publisher's note Springer Nature remains neutral with regard to jurisdictional claims in published maps and institutional affiliations.

\section{References}

1. Schmidt-Hieber M, Silling G, Schalk E, Heinz W, Panse J, Penack $\mathrm{O}$, et al. CNS infections in patients with hematological disorders (including allogeneic stem-cell transplantation)_ guidelines of the Infectious Diseases Working Party (AGIHO) of the German Society of Hematology and Medical Oncology (DGHO). Ann Oncol. 2016;27:1207-25.

2. Siegal D, Keller A, Xu W, Bhuta S, Kim DH, Kuruvilla J, et al. Central nervous system complications after allogeneic hematopoietic stem cell transplantation: incidence, manifestations, and clinical significance. Biol Blood Marrow Transplant. 2007;13:1369-79.

3. Rosello AB, Bataller L, Lorenzo I, Jarque I, Salavert M, González $\mathrm{E}$, et al. Infections of the central nervous system after unrelated donor umbilical cord blood transplantation or human leukocyte antigen-matched sibling transplantation. Biol Blood Marrow Transplant. 2017;23:134-9.

4. Schmidt-Hieber M, Schwender J, Heinz WJ, Zabelina T, Kühl JS, Mousset S, et al. Viral encephalitis after allogeneic stem cell transplantation: a rare complication with distinct characteristics of different causative agents. Haematologica. 2011;96:142-9.

5. Bleggi-Torres LF, de Medeiros BC, Werner B, Neto JZ, Loddo G, Pasquini R, et al. Neuropathological findings after bone marrow transplantation: an autopsy study of 180 cases. Bone Marrow Transplant. 2000;25:301-7.

6. Maschke M, Dietrich U, Prumbaum M, Kastrup O, Turowski B, Schaefer UW, et al. Opportunistic CNS infection after bone marrow transplantation. Bone Marrow Transplant. 1999;23: 1167-76.

7. Teive H, Carsten AL, Iwamoto FM, Almeida SM, Munhoz RP, Werneck LC, et al. Fungal encephalitis following bone marrow transplantation: clinical findings and prognosis. J Postgrad Med. 2008;54:203-5.

8. Weber C, Schaper J, Tibussek D, Adams O, Mackenzie CR, Dilloo D, et al. Diagnostic and therapeutic implications of neurological complications following paediatric haematopoietic stem cell transplantation. Bone Marrow Transplant. 2008;41:253-9.

9. Jantunen E, Volin L, Salonen O, Piilonen A, Parkkali T, Anttila VJ, et al. Central nervous system aspergillosis in allogeneic stem cell transplant recipients. Bone Marrow Transplant. 2003; 31:191-6.

10. de Medeiros BC, de Medeiros CR, Werner B, Neto JZ, Loddo G, Pasquini R, et al. Central nervous system infections following bone marrow transplantation: an autopsy report of 27 cases. J Hematotherapy Stem Cell Res. 2000;9:535-40.

11. Denier C, Bourhis JH, Lacroix C, Koscielny S, Bosq J, Sigal R, et al. Spectrum and prognosis of neurologic complications after hematopoietic transplantation. Neurology. 2006;67:1990-7.

12. Hanajiri R, Kobayashi T, Yoshioka K, Watanabe D, Watakabe K, Murata $\mathrm{Y}$, et al. Central nervous system infection following allogeneic hematopoietic stem cell transplantation. Hematol Oncol Stem Cell Ther. 2017;10:22-8.

13. Roemer E, Blau IW, Basara N, Kiehl MG, Bischoff M, Günzelmann $\mathrm{S}$, et al. Toxoplasmosis, a severe complication in allogeneic hematopoietic stem cell transplantation: successful treatment strategies during a 5-year single-center experience. Clin Infect Dis. 2001;32:E1-8.

14. Hagensee ME, Bauwens JE, Kjos B, Bowden RA. Brain abscess following marrow transplantation: experience at the Fred Hutchinson Cancer Research Center, 1984-1992. Clin Infect Dis. 1994;19:402-8.

15. Glaser CA, Honarmand S, Anderson LJ, Schnurr DP, Forghani B, Cossen CK, et al. Beyond viruses: clinical profiles and etiologies associated with encephalitis. Clin Infect Dis. 2006;43:1565-77.

16. Kang JM, Kim YJ, Kim JY, Cho EJ, Lee JH, Lee MH, et al. Neurologic complications after allogeneic hematopoietic stem cell transplantation in children: analysis of prognostic factors. Biol Blood Marrow Transplant. 2015;21:1091-8.

17. Zhang XH, Zhang JM, Han W, Chen H, Chen YH, Wang FR, et al. Viral encephalitis after haplo-identical hematopoietic stem cell transplantation: Causative viral spectrum, characteristics, and risk factors. Eur J Haematol. 2017;98:450-8.

18. Tunkel AR, Glaser CA, Bloch KC, Sejvar JJ, Marra CM, Roos $\mathrm{KL}$, et al. The management of encephalitis: clinical practice guidelines by the Infectious Diseases Society of America. Clin Infect Dis. 2008;47:303-27.

19. Lin R, Liu Q. Diagnosis and treatment of viral diseases in recipients of allogeneic hematopoietic stem cell transplantation. J Hematol Oncol. 2013;6:94.

20. Miao Q, Ma Y, Wang Q, Pan J, Zhang Y, Jin W, et al. Microbiological diagnostic performance of metagenomic nextgeneration sequencing when applied to clinical practice. Clin Infect Dis. 2018;67:S231-s40.

21. Wilson MR, Sample HA, Zorn KC, Arevalo S, Yu G, Neuhaus J, et al. Clinical metagenomic sequencing for diagnosis of meningitis and encephalitis. N Engl J Med. 2019;380:2327-40.

22. Liu Q, Xuan L, Liu H, Huang F, Zhou H, Fan Z, et al. Molecular monitoring and stepwise preemptive therapy for Epstein-Barr 
virus viremia after allogeneic stem cell transplantation. Am J Hematol. 2013;88:550-5.

23. Lin R, Wang Y, Huang F, Fan Z, Zhang S, Yang T, et al. Two dose levels of rabbit antithymocyte globulin as graft-versus-host disease prophylaxis in haploidentical stem cell transplantation: a multicenter randomized study. BMC Med. 2019;17:156.

24. Liao H, Yang Z, Yang C, Tang Y, Liu S, Guan W, et al. Impact of viral infection on acute exacerbation of asthma in out-patient clinics: a prospective study. J Thorac Dis. 2016;8:505-12.

25. Wang S, Ai J, Cui P, Zhu Y, Wu H, Zhang W. Diagnostic value and clinical application of next-generation sequencing for infections in immunosuppressed patients with corticosteroid therapy. Ann Transl Med. 2020;8:227.

26. Miller S, Naccache SN, Samayoa E, Messacar K, Arevalo S, Federman S, et al. Laboratory validation of a clinical metagenomic sequencing assay for pathogen detection in cerebrospinal fluid. Genome Res. 2019;29:831-42.

27. Wu M, Huang F, Jiang X, Fan Z, Zhou H, Liu C, et al. Herpesvirus-associated central nervous system diseases after allogeneic hematopoietic stem cell transplantation. PLoS ONE. 2013;8:e77805.

28. Liu L, Liu Q, Feng S. Management of Epstein-Barr virus-related post-transplant lymphoproliferative disorder after allogeneic hematopoietic stem cell transplantation. Therapeutic Adv Hematol. 2020;11:2040620720910964.

29. Colombo AA, Marchioni E, Diamanti L, Di Matteo AM, Baldanti F, Furione M, et al. Neurological complications involving the central nervous system after allogeneic hematopoietic stem cell transplantation during a period of evolution in transplant modalities: a cohort analysis. Transplantation. 2017;101:616-23.

30. Barba P, Piñana JL, Valcárcel D, Querol L, Martino R, Sureda A, et al. Early and late neurological complications after reducedintensity conditioning allogeneic stem cell transplantation. Biol Blood Marrow Transplant. 2009;15:1439-46.

31. Teive HA, Funke V, Bitencourt MA, de Oliveira MM, Bonfim C, Zanis-Neto J, et al. Neurological complications of hematopoietic stem cell transplantation (HSCT): a retrospective study in a HSCT center in Brazil. Arquivos de Neuro-Psiquiatria. 2008;66:685-90.

32. Abidi MZ, Hari P, Chen M, Kim S, Battiwala M, Dahi PB, et al. Virus detection in the cerebrospinal fluid of hematopoietic stem cell transplant recipients is associated with poor patient outcomes: a CIBMTR contemporary longitudinal study. Bone Marrow Transplant. 2019;54:1354-60.

33. Kawabe S, Ito Y, Ohta R, Sofue A, Gotoh K, Morishima T, et al. Comparison of the levels of human herpesvirus 6 (HHV-6) DNA and cytokines in the cerebrospinal fluid and serum of children with HHV-6 encephalopathy. J Med Virol. 2010;82:1410-5.

34. Grahn A, Bergström T, Runesson J, Studahl M. Varicella-zoster virus (VZV) DNA in serum of patients with VZV central nervous system infections. J Infect. 2016;73:254-60.

35. Vu T, Carrum G, Hutton G, Heslop HE, Brenner MK, Kamble R. Human herpesvirus- 6 encephalitis following allogeneic hematopoietic stem cell transplantation. Bone Marrow Transplant. 2007;39:705-9. 